\title{
Physiological Role of the Association Complexes of $\alpha$ - Crystallin and Its Substrates on the Chaperone Activity
}

\author{
J iahn-Shing Lee,* Tatsuya Samejima,† J iahn-Haur Liao,‡ \\ Shih-Hsiung Wu, $\neq$ and Shyh-Horng Chiou ${ }^{\mathbb{1}} \neq{ }^{1}$ \\ *Department of Ophthalmology, Chang-Gung Memorial Hospital; łLaboratory of Crystallin Research, \\ Institute of Biochemical Sciences, National Taiwan University; "I nstitute of Biological Chemistry, \\ P.O. Box 23-106, Academia, Taipei, Taiwan; and †Department of Chemistry, College \\ of Science and Engineering, Aoyama Gakuin University, Tokyo, J apan
}

Received J anuary 24, 1998

Previous reports on the chaperone activity of $\alpha$-crystallin to prevent protein denaturation and thermal aggregation have suggested that partially denatured proteins can bind $\alpha$-crystallin in its central region. Likewise, $\beta$ - and $\gamma$-crystallin can also be localized to the central cavity of $\alpha$-crystallin particle in vivo, which provides indirect evidence that $\alpha$-crystallin can function as a chaperone in the intact lens. In this study, we have further demonstrated that the binding of the substrate proteins to $\alpha$-crystallin by short-term preincubation may mimic the in vivo conditions of crystallin association. Preheating of $\alpha$-crystallin with its substrate proteins at $60^{\circ} \mathrm{C}$ for $20 \mathrm{~min}$ resulted in the formation of stable complexes between $\alpha$-crystallin and its substrates $(8.0 \%$ of insulin or $5.3 \%$ of $\gamma$-crystallin was involved in complex formation). Under such conditions, the chaperone activity of $\alpha$-crystallin to inhibit dithiothreitol-, ultraviolet-, or oxidation-induced protein aggregation can be greatly enhanced. Since UVirradiation and oxidative stress are common insults to eye lenses under normal physiological conditions, the presence of $\alpha / \gamma$ and $\alpha / \beta$ complex in vivo may play an important role to maintain the lens in a transparent state. 1998 Academic Press

$\alpha$-Crystallin, the major protein of the ocular lens, is believed to play a prominent role in the maintenance of the transparency and refractive properties of thelens $(1,2)$. It belongs to the family of small heat-shock proteins (3), consisting of two types of homologous subunits to form a dynamic multimeric complex (4). In vitro, it can function like a molecular chaperone to prevent thermal aggregation of a number of enzymes and

\footnotetext{
${ }^{1}$ To whom correspondence should be addressed. Fax: (886)-23635038; E-mail: shchiou @bc.ntu.edu.TW.
}

other lens structural proteins such as $\beta$ - and $\gamma$-crystallins (5). The mechanism of this protection may involve preferential binding of the partially denatured protein to $\alpha$-crystallin complex via hydrophobic interaction (6-8).

Whether the chaperone-like activity of $\alpha$-crystallin has any functional significance in the eye lens remains conjectural and speculative. $\alpha$-Crystallin was shown to inhibit UV-induced protein aggregation with a chaperonin effect analogous to that seen in thermal aggregation experiments (9). It can also decrease the light scattering and thiol oxidation of other crystallins under conditions of various oxidative stress (10). Because UVirradiation and oxidative stress are thought to be present under normal physiological conditions and are considered as two major risk factors for cataractogenesis $(11,12)$, it is conceivable that $\alpha$-crystallin may therefore contribute to some mechanisms that maintain the lens in a transparent state in vivo.

However, the protective ability of $\alpha$-crystallin, in terms of stoichiometry, is much lowered in UV-irradiation and oxidative stress than in thermal aggregation experiments $(1,9,10)$. Upon heating, $\alpha$-crystallin may undergo an irreversible conformational transition, with a marked increase in surface hydrophobicity, which correlates well with its increased chaperone activity $(8,13)$. Up to now, we do not know whether such structural changes actually occur under in vivo conditions. Nevertheless, it was demonstrated that, by immunoelectron microscopy, part of $\alpha$-crystallin of water soluble lens proteins contained either $\gamma$ - or $\beta$-crystallin, bound to the putative chaperone binding site in the central region of the high-molecular-weight $\alpha$-crystallin complexes $(14,15)$. This binding is similar to that seen in vitro when $\alpha$-crystallin is incubated in the presence of partially denatured forms of $\gamma$-crystallin (16).

The presence of the $\alpha / \gamma$ and $\alpha / \beta$ complexes in vivo might provide indirect evidence that $\alpha$-crystallin does 
play a functional role as a molecular chaperone in the intact lens (14). In this paper, we further demonstrate that in vitro short-term preincubation of substrate proteins and $\alpha$-crystallin engenders the crystallin complexes similar to those observed under physiological conditions. The protective abilities of $\alpha$-crystallin to inhibit protein denaturation and aggregation associated with dithiothreitol (DTT)-reduction, UV-irradiation, or $\mathrm{H}_{2} \mathrm{O}_{2}$-oxidation are found to enhance greatly. Probably through this association mechanism, $\alpha$-crystallin can provide more protection for other lens proteins against various stress under physiological conditions $(9,10)$.

\section{MATERIALS AND METHODS}

Lens crystallins were isolated from extracts of bovine lenses using a TSK HW-55(S) gel permeation column as described previously (17). The concentration of lens protein was determined by absorbance measurements using extinction coefficients of 0.83 and 2.26 at 280 $\mathrm{nm}$ for $\alpha$ - and $\gamma$-crystallins, respectively (based on $1 \mathrm{mg} / \mathrm{ml}$ ). Dithiothreitol (DTT) was purchased from Research Organics, Inc. (CleveIand, Ohio). Insulin and other chemicals were of the highest quality and purchased from Sigma Chemical Co. (St. Louis, MO).

\section{Chaperone Activity Assay for DTT-Induced Aggregation of Insulin B Chain}

Chaperone activity of $\alpha$-crystallin under DTT reduction was measured as described previously (8). This assay measures the ability of $\alpha$-crystallin to protect against the aggregation of insulin B chain in the presence of DTT. Briefly, $0.2 \mathrm{mg}$ of insulin in $1 \mathrm{ml}$ of $0.1 \mathrm{M}$ phosphate buffer, pH 7.0 (in the absence or presence of $0.2 \mathrm{mg}$ of $\alpha$ crystallin) was reduced with $20 \mathrm{mM}$ freshly prepared DTT and the kinetics of $B$ chain aggregation was followed by measuring the apparent absorption at $360 \mathrm{~nm}$ due to light scattering or turbidity formation. The measurements were performed at room temperature using a J asco 7800 UVNIS spectrophotometer and a $1 \mathrm{~cm}$ pathlength cuvette. Four different conditions were compared in this assay, which include: 1 . insulin only, 2. insulin and native $\alpha$-crystallin, 3 . insulin and preheated $\alpha$-crystallin, 4 . both insulin and $\alpha$-crystallin were preheated. The preheated samples were carried out at $60^{\circ} \mathrm{C}$ for $20 \mathrm{~min}$ and then allowed to equilibrate at room temperature for $12 \mathrm{~h}$ prior to experiments.

\section{Gel-Permeation HPLC for Analysis of Substrate Binding}

A J asco Familic-300S HPLC pump, a UVIDEC-100V detector, and a Hitachi D2500 Chromato-I ntegrator were used in conjunction with a SynChropak GPC $300(250 \times 4.6 \mathrm{~mm})$ size exclusion column for analysis of protein interaction with $\alpha$-crystallin. The mobile phase was $0.1 \mathrm{M}$ sodium phosphate $\mathrm{pH} 7.0$ with an isocratic flow rate of $0.25 \mathrm{ml} / \mathrm{min}$. Absorbance was monitored at $280 \mathrm{~nm}$. $\alpha$-Crystallin was mixed with insulin or $\gamma$-crystallin and analyzed at room temperature before and after incubation at $60^{\circ} \mathrm{C}$ for $20 \mathrm{~min}$. The samples after thermal incubation were also allowed to equilibrate at room temperature for at least $12 \mathrm{~h}$. The elution profiles of mixtures with or without heating were compared regarding relative changes in area for each peak.

\section{Chaperone Activity Assay for UV-Induced Aggregation of $\gamma$-Crystallin}

Chaperone activity of $\alpha$-crystallin under UV-irradiation was measured as described previously (18). This assay measures the ability of

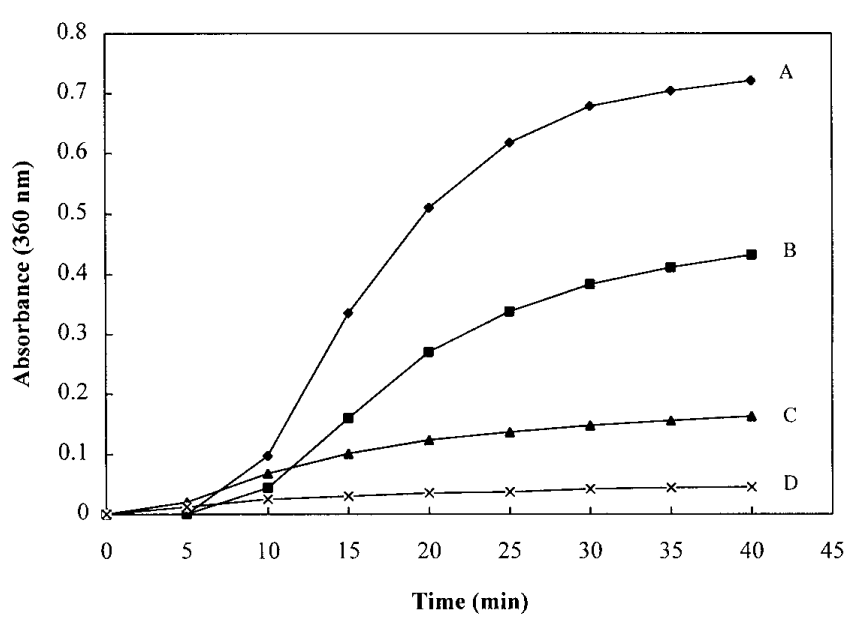

FIG. 1. DTT-induced aggregation of insulin B chains at room temperature in the absence and presence of $\alpha$-crystallin under native and preheating conditions. (A) insulin B chains with DTT in the absence of $\alpha$-crystallin; (B) insulin B chains with DTT in the presence of native $\alpha$-crystallin; (C) insulin B chains with DTT in the presence of $\alpha$-crystallin preincubated at $60^{\circ} \mathrm{C}$ for $20 \mathrm{~min}$; and (D) both insulin B chains and $\alpha$-crystallin preincubated at $60^{\circ} \mathrm{C}$ for 20 min before assays.

$\alpha$-crystallin to inhibit the aggregation of UV-irradiated $\gamma$-crystallin. Briefly, $0.2 \mathrm{mg}$ of $\gamma$-crystallin, in $1 \mathrm{ml}$ of $0.1 \mathrm{M}$ phosphate buffer, $\mathrm{pH}$ 7.0 (in the absence or presence of $0.2 \mathrm{mg}$ of $\alpha$-crystallin), was irradiated at $295 \mathrm{~nm}$ with the excitation bandpass of $20 \mathrm{~nm}$ for a fixed time period in a J asco-F P777 spectrofluorometer, and after irradiation the excitation and emission of the monochrometer were set at $600 \mathrm{~nm}$ with a bandpass of $1.5 \mathrm{~nm}$ to measure the relative scattering. Four different conditions were compared in this assay, which include: 1. $\gamma$-crystallin only, 2. $\gamma$-crystallin and native $\alpha$-crystallin, 3. $\gamma$-crystallin and preheated $\alpha$-crystallin, 4 . both $\gamma$ - and $\alpha$-crystallin were preheated. The preheated samples were carried out at $60^{\circ} \mathrm{C}$ for 20 min and then allowed to equilibrate at room temperature for $12 \mathrm{~h}$ prior to experiments.

\section{Chaperone Activity Assay for Oxidation-Induced Aggregation of $\gamma$-Crystallin}

Chaperone activity of $\alpha$-crystallin under oxidative stress was measured similar to a previous report (10). This assay measures the ability of $\alpha$-crystallin to protect against the aggregation of $\gamma$-crystallin under oxidation. Briefly, $0.5 \mathrm{mg}$ of $\gamma$-crystallin in $1 \mathrm{ml}$ of 0.1 $\mathrm{M}$ phosphate buffer, pH 7.0 (in the absence or presence of $0.5 \mathrm{mg}$ of $\alpha$-crystallin) was incubated with $0.5 \mathrm{mM}$ sodium ascorbate, $0.1 \mathrm{mM}$ $\mathrm{FeCl}_{3}, 0.3 \mathrm{mM}$ EDTA, and $1 \mathrm{mM} \mathrm{H} \mathrm{H}_{2}$. The kinetics of $\gamma$-crystallin aggregation was followed by measuring the apparent absorption at $360 \mathrm{~nm}$ due to light scattering (similar to that used in assays for DTTinduced aggregation). F our different assay conditions were compared similar to those described for UV-induced aggregation of $\gamma$-crystallin.

\section{RESULT}

In Fig. 1, upon reducing the disulfide bond between the two chains of insulin with reducing agent DTT, the $B$ chain of insulin aggregates and precipitates out (curve A), leaving the A chain in solution (8). Also shown in curve B of Fig. 1, this process can be suppressed by $\alpha$-crystallin which presumably binds the ag- 
gregation-prone protein (19). The extent of the protection for B chain from aggregation depends on the relative concentration of insulin and the chaperone, with a complete prevention of aggregation at a weight ratio of $\alpha$-crystallin to insulin of more than 6:1 (19). In Fig. 1 (curve C), the preheated $\alpha$-crystallin was shown to be more potent in suppressing theaggregation of insulin B chain than the native one. This increased chaperone activity of the preheated $\alpha$-crystallin was explained previously by the increase in its surface hydrophobicity as assessed by the ANS (1-anilino-8-naphthalene sulfonate) binding assay $(8,17,20)$. In the present study, we have further found that if both insulin and $\alpha$-crystallin were preincubated at the same condition as that of preheated $\alpha$-crystallin, the extent of apparent absorption due to light scattering would be much more depressed (curve D).

Preincubation of both insulin and $\alpha$-crystallin may lead to heat-induced interaction between these two proteins and thermal perturbation upon either one respectively. The temperature-induced changes of insulin or $\alpha$-crystallin alone can not explain the difference between curves C and D. In order to explore the interaction of insulin and $\alpha$-crystallin under thermal incubation, we have thus performed and compared gel-permeation HPLC of these two proteins before and after heating.

The gel-permeation HPLC analysis of the mixture of $\alpha$-crystallin $(0.2 \mathrm{mg} / \mathrm{ml})$ and insulin $(0.2 \mathrm{mg} / \mathrm{ml})$ is shown in Fig. 2A. Similar result of the elution profile for the mixture of $\alpha$-crystallin $(0.5 \mathrm{mg} / \mathrm{ml})$ and $\gamma$-crystallin $(0.5 \mathrm{mg} / \mathrm{ml})$ is shown in Fig. 2B. Previously $\alpha-$ crystallin was shown to act as a chaperone interacting with other proteins to prevent heat-induced protein insolubilization (5). Both mixtures consisting of the studied proteins before and after incubation at $60^{\circ} \mathrm{C}$ for 20 min respectively, showed little change in light scattering and no apparent pellet formation after centrifugation. However, comparison of the elution profiles of the mixtures clearly indicate that there is complex formation between $\alpha$-crystallin and insulin or $\gamma$-crystallin after heating. The patterns of unheated mixtures (solid lines in Fig. 2) give the identical elution profiles as those based on independent runs with each individual protein alone. However after heating at $60^{\circ} \mathrm{C}$ for 20 min (dot lines in Fig. 2), the elution time of the first peaks decrease and the area increases, indicating the formation of complex between native $\alpha$-crystallin and substrate protein. The areas of the second peaks decrease by about $8.0 \%$ and $5.3 \%$ for insulin and $\gamma$-crystallin respectively.

The short-term incubation of protein mixtures cre ates a condition that part of the aggregation-prone substrates are bound to $\alpha$-crystallin, which mimics the in vivo condition of $\alpha$-crystallin. To test whether such enhanced chaperone-like activity under DTT-induced aggregation experiments is also present under physiologi-
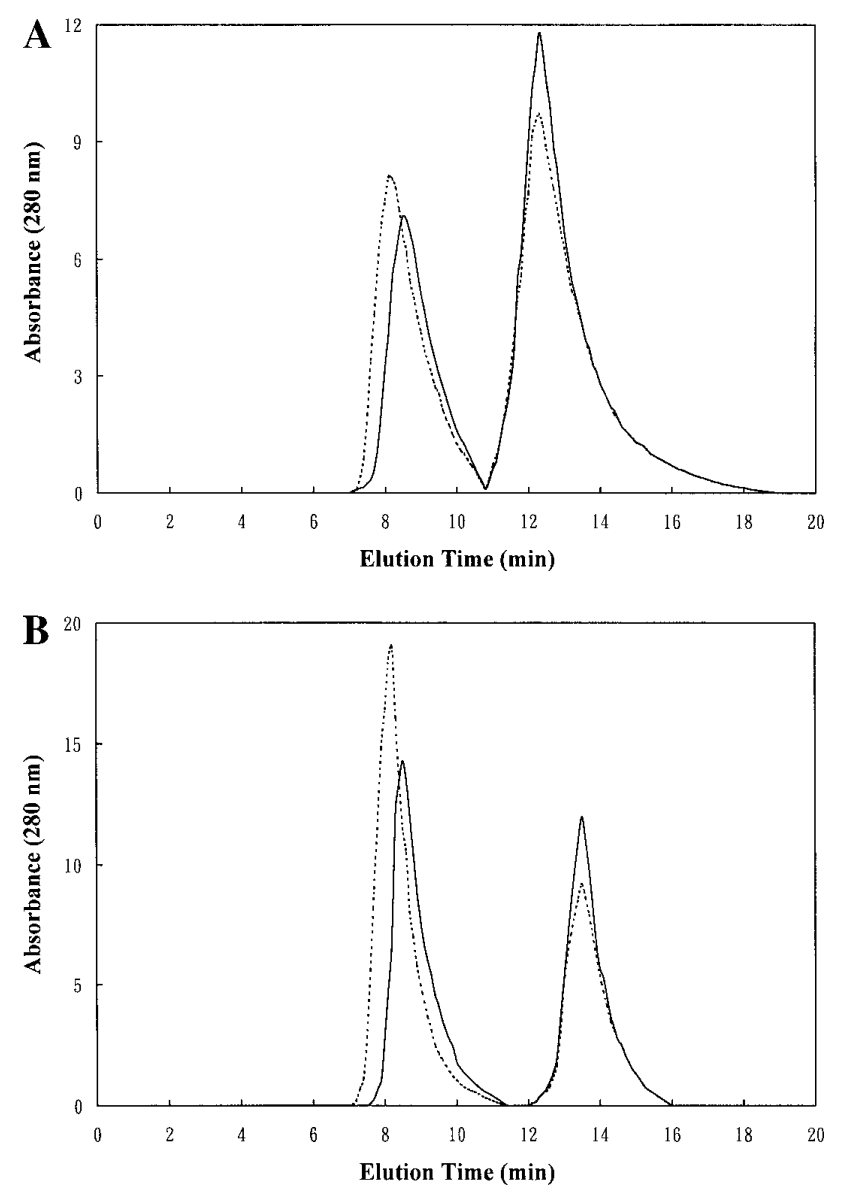

FIG. 2. Gel permeation HPLC of $\alpha$-crystallin and substrate proteins, (A) $\alpha$-crystallin plus insulin, (B) $\alpha$-crystallin plus $\gamma$-crystallin. The sample proteins were preheated at $60^{\circ} \mathrm{C}$ for $20 \mathrm{~min}$ (dotted line) and without preheating (solid line).

cal stress, we have performed similar experiments against UV-irradiation and $\mathrm{H}_{2} \mathrm{O}_{2}$-oxidation using $\gamma$ crystallin, instead of insulin, as substrate. In Fig. 3, UV-irradiation of $\gamma$-crystallin leads to aggregation and this process can be suppressed by $\alpha$-crystallin (compare curves A and B of Fig. 3). Similar to previous reports $(13,17)$, the preheated $\alpha$-crystallin exhibits a higher chaperone activity than the native one to protect $\gamma$ crystallin against photoaggregation (curve $\mathrm{C}$ of $\mathrm{Fig}$. 3). However, if the preincubation included both $\alpha$-crystallin and $\gamma$-crystallin, the extent of UV-induced protein aggregation decreased most (curve D of Fig. 3).

In Fig. 4, we have also confirmed a previous report (10) that $\alpha$-crystallin can act as a chaperone under oxidative stress, decreasing the light scattering of $\gamma$-crystallin (curves A and B of Fig. 4). F urthermore, the preheated $\alpha$-crystallin showed a higher ability to protect $\gamma$-crystallin against oxidative stress (curve $C$ of Fig. 4) even though the effect is not so prominent like that under UV-irradiation (curve C of Fig. 3). Wehave again demonstrated that the chaperone activity, in terms of 


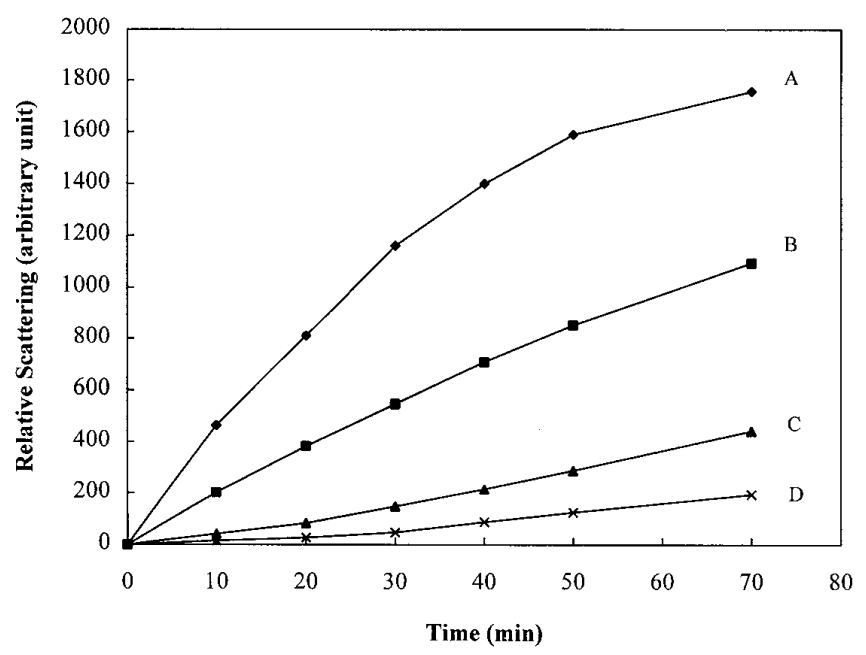

FIG. 3. UV-irradiated aggregation of $\gamma$-crystallin at room temperature in the absence and presence of $\alpha$-crystallin under native and preheating conditions. (A) UV-irradiation of $\gamma$-crystallin in the absence of $\alpha$-crystallin; (B) UV-irradiation of $\gamma$-crystallin in the presence of native $\alpha$-crystallin; (C) UV-irradiation of $\gamma$-crystallin in the presence of $\alpha$-crystallin preincubated at $60^{\circ} \mathrm{C}$ for $20 \mathrm{~min}$; and (D) both $\gamma$-crystallin and $\alpha$-crystallin preincubated at $60^{\circ} \mathrm{C}$ for $20 \mathrm{~min}$ before assays.

its ability to preserve solution transparency, is the highest (curve D of Fig. 4) when both $\alpha$-crystallin and $\gamma$-crystallin are preincubated at $60^{\circ} \mathrm{C}$ for $20 \mathrm{~min}$.

\section{DISCUSSION}

There is a considerable interest in the functional role of $\alpha$-crystallin, especially after it was shown to have in vitro chaperone activity (5). However, the mechanism of this chaperone action of $\alpha$-crystallin in the intact lens is still not very clear. Previous heat denaturation assays $(16,21)$ have demonstrated that $\alpha$-crystallin can preferentially bind to partially denatured proteins via a central region of the complex. In this process, the polypeptide is protected against further denaturation (16). In vivo, $\beta$ - and $\gamma$-crystallins can also be localized to the central region of the $\alpha$-crystallin particle, strongly suggesting that the $\alpha$-crystallin can function as a molecular chaperone in the intact lens (14). It was estimated that 3.3\% and 3.7\% of $\alpha$-crystallin in lens soluble proteins was bound by $\beta$ - and $\gamma$-crystallin respectively, and a higher percentage of binding (7.8\% and 9.0\%) was noted in the high-molecular-weight aggregate $(\mathrm{HMW}-\alpha)$ fraction (15).

However, it was reported that binding of partially denatured protein to $\alpha$-crystallin particles results in a subsequent decrease in the ability of the $\alpha$-crystallin to protect against further protein denaturation (22). In that study, a constant amount of $\alpha$-crystallin was preincubated with increasing amounts of $\gamma$-crystallin, and the resulting complexes were tested for their abil- ity to protect against heat-induced aggregation of other proteins. It is apparent that the limited amounts of binding site may enable only a fixed number of partially denatured proteins to bind to the $\alpha$-crystallin particles. In the present study, using a fixed number of substrate proteins, we have otherwise demonstrated that prebinding of substrate proteins to $\alpha$-crystallin showed higher chaperone efficiency against various types of insults. It is possible that the entrapped substrate proteins become more resistant to UV-irradiated and oxidative stress or the decreased concentration of the unbound aggregation-prone peptides makes the stressinduced aggregation less prominent.

Although the binding pattern of $\beta$ - or $\gamma$-arystallin to $\alpha$-crystallin in vivo may be similar to that of in vitro by thermal incubation, it is physiologically impossible that such high temperature $\left(60^{\circ} \mathrm{C}\right)$ exist in vivo to account for the formation of $\alpha / \gamma$ and $\alpha / \beta$ complexes in the intact lens. One of the alternative mechanisms to account for these complex formation is mediated by UV-irradiation. $\alpha$ Crystallin can bind with its substrate under UV-irradiation, however the reaction intermediates are different in UV-irradiated versus heat-induced aggregation. UV irradiation was shown to generate covalently linked aggregates which were not dissociated by SDS and reducing agents, whereas heat-induced aggregates could be easily dissociated under the same conditions (9). Another possibility for the formation of these complexes is through the process of oxidative stress. However, the interaction of $\alpha /$ $\gamma$ complex formed from in vitro oxidation was too weak to be isolated with procedures used to obtain the thermally induced complexes (10).

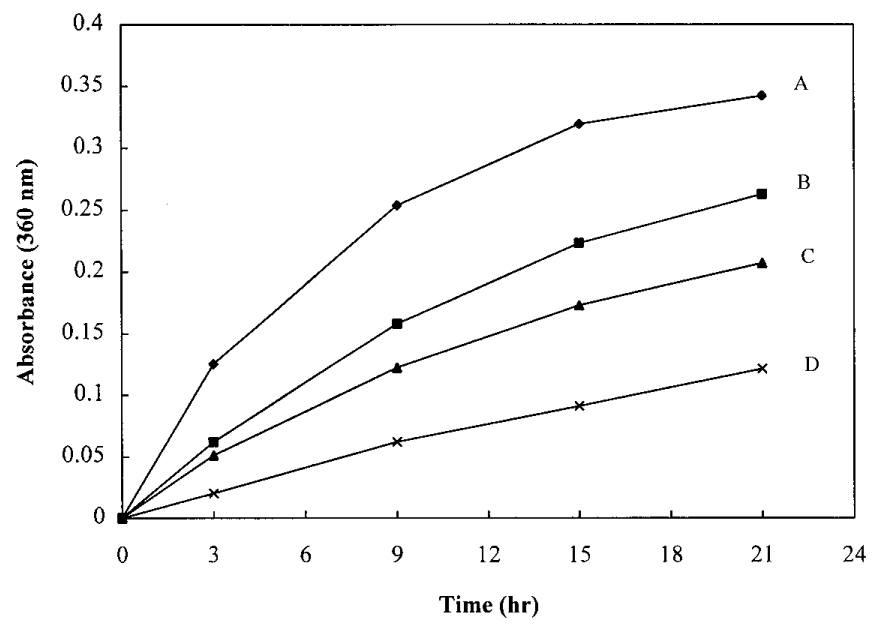

FIG. 4. Oxidation-induced aggregation of $\gamma$-crystallin at room temperature in the absence and presence of $\alpha$-crystallin under native and preheating conditions. (A) oxidation-induced aggregation of $\gamma$ crystallin in the absence of $\alpha$-crystallin; (B) oxidation-induced aggregation of $\gamma$-crystallin in the presence of native $\alpha$-crystallin; (C) oxidation-induced aggregation of $\gamma$-crystallin in the presence of $\alpha$-crystallin preincubated at $60^{\circ} \mathrm{C}$ for $20 \mathrm{~min}$; and (D) both $\gamma$-crystallin and $\alpha$-crystallin preincubated at $60^{\circ} \mathrm{C}$ for $20 \mathrm{~min}$ before assays. 
It remains enigmatic how $\alpha / \gamma$ and $\alpha / \beta$ complexes are formed in vivo. However as shown in this study the formation of such complexes may afford higher efficacies of protection to preserve the ocular lens in a transparent state against various physiological stress. Since the ocular lens is constantly exposed to visible and UV light irradiation and there is a significant amount of $\mathrm{H}_{2} \mathrm{O}_{2}(30 \mu \mathrm{M})$ in the aqueous humor of the normal human eye (23), it is reasonable to assume that an efficient chaperonelike protective action against photochemical and oxidative insults indeed exists in vivo. In this context $\alpha$-crystallin may be the best candidate for mediating such a protective role in the lens because the molecular chaperone activity of $\alpha$-crystallin have been correlated with the extents of posttranslational modifications, such as UV-irradiation (17) and oxidation (24).

In this study, we did not include $\beta$-crystallin as a substrate, mainly because its oxidation at room temperature produced only a slight increase in light scattering, in contrast to those observations obtained with $\gamma$-crystallin (10). On the other hand, the preincubation under higher temperatures and/or for longer periods may increase the amounts of complex formation be tween $\alpha$-crystallin and its substrates. However, we have found that $\alpha$-crystallin could not provide longterm heating ( $>30 \mathrm{~min}$ ) protection against thermally induced aggregation of $\gamma$-crystallin, and $\alpha / \gamma$ complex may gradually become insoluble at temperatures higher than $60^{\circ} \mathrm{C}$. Nevertheless, it is interesting to note that such short-term preincubation as shown in this study gave the percentage of $\alpha / \gamma$ complex formation very close to that seen in vivo (15).

In conclusion, we have demonstrated that the preincubation of $\gamma$ - and $\alpha$-crystallin mixtures at $60^{\circ} \mathrm{C}$ for 20 min resulted in the formation of $\alpha / \gamma$ complex, which may mimic the formation of such complexes in vivo. Under such conditions of complex formation, the chaperone-like activity of $\alpha$-crystallin against UV-irradiation or $\mathrm{H}_{2} \mathrm{O}_{2}$-induced oxidation on lens proteins was shown to be more effective than that of native or preheated $\alpha$-crystallin. It is apparent that in addition to the inherent chaperone activity of $\alpha$-crystallin, some other factors which induce the formation of $\alpha / \gamma$ and $\alpha /$ $\beta$ complexes in vivo may also work in a cooperative manner to protect the lens against physiological stress.

\section{ACKNOWLEDGMENTS}

This work was supported by Academia Sinica and the National Science Council (NSC Grants 83-0203-B-001-086, 83-0418-B-001020BA, 84-2311-B-001-050-BA, and 86-2311-B-002-031-B15), Taipei, Taiwan.

\section{REFERENCES}

1. Horwitz, J. (1993) Invest. Ophthalmol. Vis. Sci. 34, 10-22.

2. Groenen, P.J . T. A., Merck, K. B., de J ong, W. W., and Bloemendal, H. (1994) Eur. J . Biochem. 225, 1- 19.

3. Ehrnsperger, M., Graber, S., Gaestel, M., and Buchner, J . (1997) Curr. Eye Res. 16, 221- 229.

4. van der Quderaa, F., de J ong, W., Hilderink, A., and Blomendal, H. (1974) Eur. J . Biochem. 49, 157- 168.

5. Horwitz, J . (1992) Proc. Natl. Acad. Sci. USA 89, 10449- 10453.

6. Boyle, D., and Takemoto, L. (1994) Exp. Eye Res. 58, 9- 16.

7. Raman, B., and Rao, C. M. (1994) J . Biol. Chem. 269, 2726427268.

8. Das, K. P., and Surewicz, W. K. (1995) FEBS Lett. 369, 321325.

9. Borkman, R. F., Knight, G., and Obi, B. (1996) Exp. EyeRes. 62, $141-148$.

10. Wang, K., and Spector, A. (1995) Invest. Ophthalmol. Vis. Sci. 36, 311- 321.

11. Spector, A., and Garner, W. H. (1981) Exp. Eye Res. 33, 673681.

12. Walker, M. L., and Borkman, R. F. (1989) Exp. EyeRes. 48, 375383.

13. Das, B. K., Liang, J J J . N., and Chakrabarti, B. (1997) Curr. Eye Res. 16, 303-309.

14. Boyle, D., and Takemoto, L. (1994) Exp. Eye Res. 58, 9- 16.

15. Takemoto, L., and Boyle, D. (1994) Curr. Eye Res. 13, 35- 44.

16. Boyle, D., Gopalakrishnan, S., and Takemoto, L. (1993) Biochem. Biophys. Res. Commun. 192, 1147-1154.

17. Lee, J.-S., Liao, J.-H., Wu, S.-H., and Chiou, S.-H. (1997) J . Protein Chem. 16, 283- 289.

18. Lee, J.-S., Satoh, T., Shinoda, H., Samejima, T., Wu, S.-H., and Chiou, S.-H. (1997) Biochem. Biophys. Res. Commun. 237, 277282.

19. Farahbakhsh, Z.T., Huang, Q. L., Ding, L. L., Altenbach, C., Steinhoff, H. J ., Horwitz, J., and Hubbell, W. L. (1995) Biochemistry 34, 509-516.

20. Raman, B., Ramakrishna, T., and Rao, C. M. (1995) FEBS Lett. 365, $133-136$.

21. Rao, P. V., Horwitz, J., and Zigler, J. S., J r. (1993) Biochem. Biophys. Res. Commun. 190, 786- 793.

22. Takemoto, L., and Boyle, D. (1994) Arch. Biochem. Biophys. 315, 133- 136.

23. Ramachandran, S., Morris, S. M., Devamanoharan, P., Henein, M., and Varma, S. D. (1991) Exp. Eys. Res. 53, 503-506.

24. Cherian, M., and Abraham, E. C. (1995) Biochem. Biophys. Res. Commun. 208, 675-679. 